\title{
Comparing integration and contextual binding accounts of memory impairment
}

\section{Pascale Gisquet-Verrier (iD and David Riccio}

In their recent Opinion (A contextual binding theory of episodic memory: systems consolidation reconsidered. Nat. Rev. Neurosci. 20, 364-375 (2019)) $)^{1}$, Yonelinas et al. reconsidered the standard systems consolidation theory (SSCT) ${ }^{2,3}$ and proposed the contextual binding theory (CBT). For these authors, the long-term development of memory and forgetting can be explained by the way information has been bound during memory formation. Information will be forgotten because different material that occurs in the same context interferes with the item to be learned. The authors propose that CBT can explain better than the SSCT several forgetting effects, including interference effects and retrograde amnesia after post-training hippocampal lesions.

We were pleased to see that this CBT view has much in common with our own 'integration concept' (IC), which we introduced to challenge the consolidation-reconsolidation hypothesis $^{4,5}$. According to the IC, memories, when in an active state (after training or reactivation), become malleable and integrate new information that is present. Depending on the information available at that time, memories can be updated, strengthened (by coherent information), disrupted (by incoherent information resulting from, for example, amnesic treatments or interference) or greatly altered (false memory). We have described evidence ${ }^{5,6}$ showing that performance disruption due to post-training amnesic treatments mainly results from impairments in retrieval that are induced by contextual differences between training and testing.

There are clear convergences between CBT and the IC model. Both accounts challenge the long-held consolidation hypotheses and emphasize a prominent role of environmental context (internal and external) as a major determinant of forgetting. Both accounts note that contextual information presented just before or just after the study event have similar effects on memory, a concept consistent with recent findings ${ }^{7-9}$.

However, some essential differences should also be noted. Although both IC and CBT claim to explain temporally graded retrograde amnesia, they do not address the same consolidation processes. IC proposes to explain retrograde amnesia resulting from amnesic treatments delivered during the first minutes following training and thus affecting presumed consolidation-reconsolidation processes. By contrast, CBT suggests an explanation for retrograde amnesia resulting from hippocampal lesions administered days to weeks after training, therefore concerning standard systems consolidation.

Another principal difference between the two frameworks concerns the origin of memory impairment. For CBT, the main source of forgetting is interference between memories that share similar context or content during memory formation. The IC account proposes that forgetting results from a contextual mismatch between acquisition and testing, inducing retrieval difficulties ${ }^{10}$. In agreement with the IC, active memories are malleable and integrate any contextual information present. Accordingly, interference is only one source of disruption, among others. As a consequence, the IC model can account for various performance modulations, such as anterograde and retrograde amnesia ${ }^{5,6}$, interference, false memories, as well as counterconditioning and promnesic effects.
Interestingly, the recent literature seems to converge towards the same aim: revisiting serious shortcomings of older theories of memory impairment. By doing that, $\mathrm{CBT}$ and IC both serve to stimulate new conceptions of memory.

Pascale Gisquet-Verrier (iD) ${ }^{*}$ and David Riccio ${ }^{2}$ IInstitut des Neurosciences Paris-Saclay (Neuro-PSI), Université Paris-Sud, CNRS UMR 9197, Université Paris-Saclay, Orsay, France. ${ }^{2}$ Department of Psychological Sciences, Kent State University, Kent, $\mathrm{OH}$, USA. *e-mail: pascale.gisquet@u-psud.fr https://doi.org/10.1038/s41583-019-0188-3

1. Yonelinas, A. P. et al. A contextual binding theory of episodic memory: systems consolidation reconsidered. Nat. Rev. Neurosci. 20, 364-375 (2019).

2. Dudai, Y., Karni, A. \& Born, J. The consolidation and transformation of memory. Neuron $\mathbf{8 8}, 20-32$ (2015).

3. Frankland, P. W. \& Bontempi, B. The organization of recent and remote memories. Nat. Rev. Neurosci. 6, 119-130 (2005)

4. Gisquet-Verrier, P. et al. Integration of new information with active memory accounts for retrograde amnesia: A challenge to the consolidation/reconsolidation hypothesis? J. Neurosci. 35, 11623-11633 (2015).

5. Gisquet-Verrier, P. \& Riccio, D. Memory integration: an alternative to the consolidation/reconsolidation hypothesis. Prog. Neurobiol. 171, 15-31 (2018).

6. Gisquet-Verrier, P. \& Riccio, D. C. Memory integration as a challenge to the consolidation/reconsolidation hypothesis: similarities, differences and perspectives. Front. Syst. Neurosci. 12, 71 (2019).

7. Cai, D. J. et al. A shared neural ensemble links distinct contextual memories encoded close in time. Nature 534, 115-118 (2016).

8. Rashid, A. J. et al. Competition between engrams influences fear memory formation and recall. Science 353, 383-387 (2016)

9. Redondo, R. L. \& Morris, R. G. M. Making memories last: the synaptic tagging and capture hypothesis. Nat. Rev. Neurosci. 12, 17-30 (2011).

10. Tulving, E. \& Thomson, D. Encoding specificity and retrieval processes in episodic memory. Psychol. Rev. 86, 739-748 (1973).

Competing interests

The authors declare no competing interests.

\section{Active and effective replay: systems consolidation reconsidered again}

\section{James W. Antony (D) and Anna C. Schapiro}

In their recent Opinion article (A contextual binding theory of episodic memory: systems consolidation reconsidered. Nat. Rev. Neurosci. 20, 364-375 (2019)) $)^{1}$, Yonelinas et al. propose that findings often taken as evidence for standard systems consolidation theory (SSCT) can be reinterpreted in a contextual binding (CB) framework. We agree that context is critical for explaining many memory phenomena and that SSCT, as defined, is probably incorrect. We do not advocate for the ideas that all memories become hippocampally independent, that the ones that do come to rely on neocortex retain the same quality or that the hippocampus does not undergo further learning with replay. Thus, on many counts, we are in agreement. However, we do think that during sleep and offline waking periods, hippocampalneocortical interactions promote active transformation of memories resulting in increased neocortical engagement, which can usefully be called 'systems consolidation', and that some key empirical findings in this area are not predicted by the $\mathrm{CB}$ framework.

The CB account posits that replay (in wake or sleep) reflects context-related residual activity, which should tend to diminish with 
changes in spatial and temporal context (absent any retrieval driving context reinstatement). But replay seems to be more persistent and adaptive than this, as it can occur as frequently for a remote spatial context as for the current environment ${ }^{2}$; has been observed 10 hours after exposure to a novel environment, with stronger activity during sleep than wake periods $^{3}$; and, critically, can occur more for infrequently experienced ${ }^{4}$, gradually learned ${ }^{5}$ and weakly encoded ${ }^{6}$ information. These findings may not be strictly inconsistent with $\mathrm{CB}$, but they are not motivated by it; additional mechanisms would be needed to explain why context is more strongly reinstated in these situations, especially during sleep.

We think there is strong evidence that sleep benefits memory beyond the reduction of contextual interference, and that this active process drives systems consolidation (as defined above). If sleep primarily benefits hippocampus-dependent memory by reducing interference or through local consolidation processes, specific active cortical events and hippocampal-cortical interactions during sleep should not be robustly and causally related to later memory. However, cortical replay coincides with hippocampal replay ${ }^{7}$ and high-frequency replay-associated bursts called ripples ${ }^{8}$, and this coupling is associated with later memory ${ }^{9}$. Hippocampal and neocortical ripples coincide and their coupling increases with learning ${ }^{10}$, and disrupting the coupling between hippocampal ripples and cortical sleep spindles impairs memory retention ${ }^{11}$. In addition, optimal replay relies on the potential for spindles to occur ${ }^{12}$, and artificially boosting individual slow oscillations increases spindle power and improves memory ${ }^{13}$. These processes seem to promote systems consolidation: timing optogenetic stimulations of the neocortex precisely to hippocampal ripples enhances endogenous hippocampal-neocortical coupling and alters neocortical neuronal spiking patterns that support behaviour ${ }^{14}$.

Yonelinas et al. argue that replay primarily reflects prior memory formation rather than driving subsequent memory transformation. However, they acknowledge that postencoding hippocampal activity may cause local cellular consolidation or re-encoding that could sometimes "lead to the formation of strong neocortical semantic representations that could support decontextualized memory for remote events". This latter mechanism fits well into our conceptualization of systems consolidation; we contend that this is a feature, rather than a side effect, of replay. Although more work is needed for full confidence in this contention - such as experiments that carefully track and manipulate the influence of the hippocampus on cortical representations ${ }^{15}$ - we think the evidence already points to replay having a critical and active role in driving consolidation across memory systems.

James W. Antony (D) $1 *$ and Anna C. Schapiro ${ }^{2 *}$ 'Princeton Neuroscience Institute, Princeton University, Princeton, NJ, USA. Department of Psychology, University of Pennsylvania, Philadelphia, PA, USA. *e-mail: jantony@princeton.edu. aschapir@sas.upenn.edu https://doi.org/10.1038/s41583-019-0191-8

1. Yonelinas, A. P. et al. A contextual binding theory of episodic memory: systems consolidation reconsidered. Nat. Rev. Neurosci. 20, 364-375 (2019).

2. Karlsson, M. P. \& Frank, L. M. Awake replay of remote experiences in the hippocampus. Nat. Neurosci. 12, 913-918 (2009)

3. Giri, B. et al. Hippocampal reactivation extends for several hours following novel experience. J. Neurosci. 39, 866-875 (2019).

4. Gupta, A. S, et al. Hippocampal replay is not a simple function of experience Neuron 65, 695-705 (2010).

5. van de Ven, G. M. et al. Hippocampal offline reactivation consolidates recently formed cell assembly patterns during sharp wave-ripples. Neuron 92, 968-974 (2016).
6. Schapiro, A. C. et al. Human hippocampal replay during rest prioritizes weakly learned information and predicts memory performance. Nat. Commun. 9 , 3920 (2018).

7. Ji, D. \& Wilson, M. A. Coordinated memory replay in the visual cortex and hippocampus during sleep. Nat. Neurosci. 10, 100-107 (2007).

8. Wilber, A. A. et al. Laminar organization of encoding and memory reactivation in the parietal cortex. Neuron 95, 1406-1419 (2017).

9. Zhang, H., Fell, J. \& Axmacher, N. Electrophysiological mechanisms of human memory consolidation. Nat. Commun. 9, 4103 (2018).

10. Khodagholy, D., Gelinas, J. N. \& Buzsáki, G. Learning-enhanced coupling between ripple oscillations in association cortices and hippocampus. Science 358, 369-372 (2017).

11. Xia, F. et al. Parvalbumin-positive interneurons mediate neocortical-hippocampal interactions that are necessary for memory consolidation. eLife 6 , e27868 (2017).

12. Antony, J. W. et al. Sleep spindle refractoriness segregates periods of memory reactivation. Curr. Biol. 28, 1736-1743 (2018).

13. Ngo, H. V. et al. Auditory closed-loop stimulation of the sleep slow oscillation enhances memory. Neuron 78, 545-553 (2013).

14. Maingret, N. et al. Hippocampo-cortical coupling mediates memory consolidation during sleep. Nat. Neurosci. 19, 959-964 (2016).

15. Mao, D. et al. Hippocampus-dependent emergence of spatial sequence coding in retrosplenial cortex. Proc. Natl Acad. Sci. USA 115, 8015-8018 (2018).

\title{
Reply to 'Active and effective replay: systems consolidation reconsidered again'
}

\author{
Andrew P. Yonelinas (1D, Charan Ranganath, Arne D. Ekstrom \\ and Brian J. Wiltgen
}

In their Correspondence article (Active and effective replay: systems consolidation reconsidered again. Nat. Rev. Neurosci. https:// doi.org/10.1038/s41583-019-0191-8 (2019))1', Antony and Schapiro agree that the concept of contextual binding (CB) described in our Opinion article (A contextual binding theory of episodic memory: systems consolidation reconsidered. Nat. Rev. Neurosci. 20, 364-375 $(2019))^{2}$ is crucial for explaining episodic memory, and that many aspects of standard systems consolidation theory are probably incorrect. They also point to some recent sleep and replay studies that might be taken as evidence for a form of systems consolidation that maintains that the hippocampus rapidly trains the cortex during offline periods of sleep or rest. Although we agree that these findings are compatible with a modified form of systems consolidation, we contend that the studies summarized by Antony and Schapiro do not necessitate such an account.

For example, demonstrations that replay correlates with subsequent memory, or that sharp wave-ripple (SWR) activity in the hippocampus correlates (in some instances) with activity in the cortex ${ }^{3}$, are consistent with other theoretical accounts such as CB. That is, because episodic memory involves hippocampal binding of item information and context information that is in the cortex, residual or potentiated encoding activity across the hippocampus and cortex should be observed after the nominal encoding event is over, even if the hippocampus is not actively training the cortex at the time. Furthermore, although experimental manipulations of SWRs in the hippocampus and/or neocortex can affect subsequent memory ${ }^{4}$, the studies performed to date do not show whether those manipulations prevented the hypothesized transfer of information from the hippocampus to the cortex, or whether they affected the hippocampal or cortical representations themselves. For instance, it would be reasonable to infer that interfering with hippocampal function during an SWR could disrupt a hippocampal (or cortico-hippocampal) memory trace, or that 